\title{
De pardos disfrazados a blancos poco claros: clasificaciones raciales en el Brasil de los siglos XVIII-XIX ${ }^{*}$
}

\author{
Jocélio Teles dos Santos** \\ Universidad Federal de Bahía, Brasil \\ jocelio@ufba.br
}

Recibido: septiembre de 2013

Aprobado: marzo de 2013

\begin{abstract}
Resumen: Este artículo analiza el sistema de clasificación racial en el Brasil de los siglos XVIII y XIX. Tiene por objeto demostrar que la clasificación colonial con base en el color puede ser visto por su diversidad y como algo constitutivo de sistemas locales de clasificaciones en consonancia y/o disonancia con la metrópoli del imperio trasatlántico. Para demostrar esta tesis se estudia la clasificación usada por la Casa de la Misericordia, institución católica secular que el imperio portugués trasplantó a sus colonias a partir del siglo xvi. El espacio de investigación es la institución señalada, creada por la Hermandad de la Santa Casa de Misericordia con el propósito de brindar refugio, apoyo y asistencia a los niños/as recién nacidos y abandonados.
\end{abstract}

Palabras claves: clasificación racial, Brasil, siglos XVIII y XIX, Santa Casa de la Misericordia, Salvador de Bahía.

\section{Of pardos disguised as white little clear: Racial classifications in Brazil of the centuries XVIII-XIX}

Abstract: This paper analyzes the system of racial classification in Brazil in the XVIII and XIX centuries. Aims to demonstrate that the colonial classification based on color can be

\footnotetext{
* Agradecemos a Kenneth Salas Olivera, estudiante del Programa de Historia de la Universidad de Cartagena, la traducción de este artículo.

** Antropólogo, magister y doctor en Antropología Social de la Universidad de São Paulo. Es profesor Asociado III de la Universidad Federal de Bahía, donde ocupó el jefe del Departamento de Antropología, el Director del Centro de Estudios Afro-Orientales y la coordinación de la maestría en estudios étnicos y africanos. Enseña en pregrado y postgrado. Âreas de investigación: religiosidad afro-brasileña; acción afirmativa en la educación superior. Editor de la revista Afro-Asia.
}

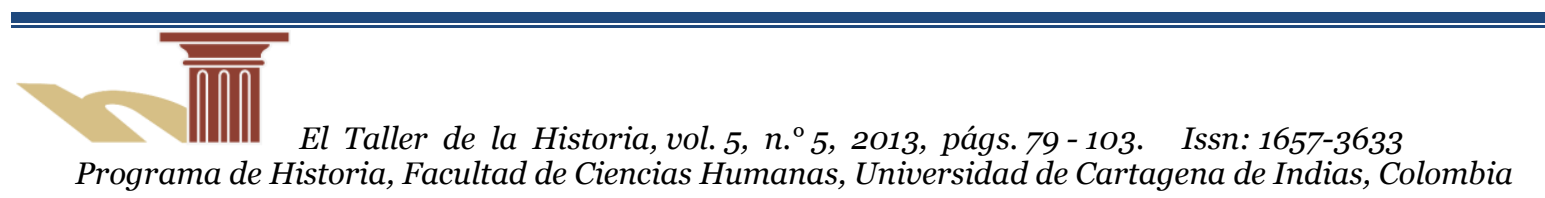


seen by its diversity as constitutive of local systems in line classifications and/or dissonance with the metropolis of transatlantic empire. To prove this thesis, the classification used by the House of Mercy Catholic secular institution that the Portuguese empire transplanted to its colonies from the sixteenth century is studied. The research area is the designated institution, created by the Brotherhood of the Holy House of Mercy in order to provide shelter, support and assistance to children/as newborns and abandoned.

Key words: racial classification, Brazil, XVIII and XIX centuries, Holy House of Mercy, Salvador de Bahía.

\section{Presentación}

El análisis sobre el sistema de clasificación racial se ha constituido en un tema candente de las ciencias sociales en Brasil. Desde la segunda mitad del siglo xx los análisis comparativos buscan analizar los matices de nuestro sistema multipolar, en el que varias categorías raciales son utilizadas en la vida cotidiana (mulato, pardo, moreno, criollo, preto, negro, moreno claro), en oposición a las del sistema norteamericano en que la clasificación tiende a una polarización, "blacks versus whites". ${ }^{1}$

En las dos últimas décadas el debate sobre las políticas de acción afirmativa en Brasil se ha desplazado del ámbito académico hacia los movimientos negros, los organismos gubernamentales y los medios de comunicación. El cuestionamiento de cómo definir las "personas de color" se convirtió en una búsqueda recurrente, en la medida en el que la clasificación en lo cotidiano asociado a las representaciones sobre mezcla "racial" se convirtió en una especie de talón de Aquiles en Brasil.

En las acaloradas discusiones académicas los argumentos apuntan el siglo diecinueve como el período de la utilización científica de la categoría de "raza" y su relación con la sociedad brasileña. El recurso usado es el del constante apoyo en la

\footnotetext{
${ }^{1}$ Ver Lívio Sansone, "Nem somente preto ou negro: o sistema de classificação racial no Brasil que muda", em Afro-Ásia n. ${ }^{\circ}$ 18, Salvador de Bahía, Universidade Federal da Bahia, 1996, pp.165-188; Carl N. Degler, Nem preto nem branco. Escravidão e relações raciais no Brasil e nos EUA, Rio de Janeiro, Labor do Brasil, 1976; Peter Fry, "O que a cinderela negra tem a dizer sobre a política racial no Brasil”, em Revista USP n. ${ }^{\circ}$ 28, São Paulo, Universidade de São Paulo, 1989, pp.122-135; Donald Pierson, Brancos e pretos na Bahia, São Paulo, Cia. Editora Nacional, 1971; Michael Hanchard, “'Americanos', brasileiros e a cor da espécie humana: uma resposta a Peter Fry”, em Revista USP n. ${ }^{\circ} 31$ São Paulo, Universidade de São Paulo, 1989, pp.164-175.
} 
historiografía, algo muy abundante en la literatura de las ciencias sociales de este país. $^{2}$

En este trabajo intento mostrar que la clasificación del color en el Brasil colonial ya se mostraba más multipolar de lo que pensamos, y que podemos visualizar la construcción de un sistema local de clasificaciones en consonancia o en disonancia con la metrópoli del imperio trasatlántico. El ámbito de la investigación es la Casa Santa de la Misericordia, institución católica secular que el imperio portugués trasladó a las colonias desde los comienzos del siglo XVI. El foco de la investigación es la Rueda de los Expósitos, un espacio creado por la hermandad para dar abrigo, refugio, amparo y asistencia a los niños recién nacidos abandonados. ${ }^{3}$

Nuestros argumentos apuntan a señalar que en estas instituciones las clasificaciones se dieron en dos momentos: el de registro y el de la confrontación de los expósitos, reflejando el uso de categorías que apuntan el ejercicio de prácticas sociales relativas a una clasificación racial durante el periodo 1763-1871. Inicialmente cuando se recogían a los niños/as, se registraban datos relativos a la fecha del recogimiento, sexo, color, y el modo como estaba vestido. Posteriormente la hermandad entregaba al niño a una nodriza, por un periodo de tres años, pagándole regularmente por la alimentación y vestuario. Algunos meses después de haber sido

\footnotetext{
${ }^{2}$ Varios son los trabajos contemporáneos que analizando problemas y periodos distintos del siglo XX, se reportan básicamente a los estudios desenvueltos sobre la segunda mitad del siglo XIX, principalmente el periodo pos-abolición. Ver por ejemplo, Giralda Seyferth, "Construindo a nação: hierarquias raciais e o papel do racismo na política de imigração e colonização", in Marcos C. Maio e Ricardo V. Santos (org.), Raça, Ciência e Sociedade, Rio de Janeiro, Fiocruz / Centro Cultural Banco do Brasil, 1996, pp.41-58; John M. Monteiro, "As 'raças' indígenas no pensamento brasileiro do império", in Maio e Santos (org.), Raça, Ciência e Sociedade, pp.15-22; Ivonne Maggie, “'Aqueles a quem foi negada a cor do dia': as categorias cor e raça na cultura brasileira”, in Maio e Santos (org.), Raça, pp.225-234; Antonio Sérgio Guimaráes, Racismo e anti-racismo no Brasil, São Paulo, Editora 34, 1999; Delcele M. Queiroz, Raça, género e educação superior, tese de doutorado, Universidade Federal da Bahia, 2001; Kim D. Butler, Freedom given. Freedoms won. AfroBrazlians in post-abolition. Sâo Paulo and Salvador, New Jersey, Rutgers University Press, 1998.

${ }^{3}$ Sobre la Santa Casa de la Misericordia, ver Carlos Ott, A Santa Casa da Misericòrdia da Cidade do Salvador, Rio de Janeiro, MEC, 1960; A. J. R. Russell-Wood, Fidalgos e Filantropos - A Santa Casa da Misericòrdia da Bahia, 1550-1755, Brasília, UnB, 1981; Neuza Rodrigues Esteves (org.), Irmàos da Santa Casa da Misericòrdia da Bahia - século XVII, Salvador, Santa Casa da Misericòrdia, 1977; Isabel dos Guimaráes Sá, A Misericòrdia da Bahia. Quando o rico se faz pobre: misericòrdias, caridade e poder no império português 1500-180o, Lisboa, Comissão Nacional para as Comemorações dos Descobrimentos Portugueses, 1997; Stuart B. Schwartz, Segredos Internos. Engenhos e escravos na sociedade colonial, São Paulo, Companhia das Letras, 1988; Paulo Segundo da Costa, Ações sociais da Santa Casa da Misericòrdia, Salvador, Contexto \& Arte, 2001; Maria Lúcia Montes, Misericòrdia, a força de um legado histórico: pesquisa realizada para a Santa Casa da Misericòrdia da Bahia, São Paulo, [s. n.], 2002.
} 
entregados a las "ama de casa", los niños retornaban para una observación de sus condiciones físicas. Es en este momento que se percibe la formación de una etnogénesis local, entendida como la construcción de una clasificación étnica-racial, la que se contrapone a la clasificación usada por la Santa Casa de la Misericordia con sede en Lisboa, hecho que muestra una continuidad de nuestras angustias clasificatorias, aun tan presentes en el inicio del siglo XXI.

\section{1.- Clasificación del color en los seiscientos y setecientos}

Desde el siglo XVII los términos "negro", "mulato" y "criollo" estaban presentes en los documentos de la Santa Casa de la Misericordia de Bahía. En testamentos de señores que dejaron significativas donaciones se observa los términos de clasificación que indica tanto la condición social como también una marca de "color". En el testamento de Belchior Fr. Queimado, otorgado el 30 de enero de 1637, se registran los esclavos "Jacinto, mulato de diecisiete años", "João, mulato avaluado en $32 \$$ reales", "Angella, mulata y su hijo de seis años, mulato llamado Lázaro y su hija de dos años, llamada Eva, todos avaluados en $35 \$$ rs" y una criolla llamada Ana. ${ }^{4}$ En el testamento de André Fernandes de Bastos y de su mujer Catherina de Azevedo, registrado el 23 de noviembre de 1652, consta que João Alves de Fonseca, propietario de hacienda y deudor de la pareja, había dejado un "escrito" donde decía tener un hijo mulatico/mulato, con una esclava catalogada de "negra". 5

Es probable que los sentidos de los dos términos fuesen los que aparecerían en el diccionario en el siglo XVIII, pues "negro", según Antonio Moraes Silva era el individuo desgraciado, triste, infausto, o aquel "de color preto como la tinta de escribir, o carbón apagado”, pero también se aplicaba al hombre negro, fuese libre o "cautivo". ${ }^{6}$ El término "mulato" clasificaba el hijo de un caballo con una burra, así como el individuo que era resultado de una relación interracial, el "hijo o hija de negro con blanca, o al revés, o de mulato con blanco hasta cierto grado".7

\footnotetext{
${ }^{4}$ Arquivo de la Santa Casa de la Misericòrdia de Bahia, Livro $1^{\circ}$ do Tombo (Escrituras, aforamentos e testamentos), 1629-1635, f.410.

${ }^{5}$ Arquivo da Santa Casa da Misericòrdia da Bahia, Livro $2^{\circ}$ do Tombo, 1652-1685, f.16

${ }^{6}$ Dicionário da Lingua Portuguesa recopilado de vocabulários impressos até agora, e nesta segunda edicäo novamente emendado e muito acrescentado por Antonio Moraes Silva, Lisboa, Typographia Lacerdina, 1813.

${ }^{7}$ Dicionário da Lingua Portuguesa.
} 
El sistema lingüístico esclavista permitía reordenamientos conceptuales y muestra una flexibilidad en el uso de categorías raciales en el Brasil colonial. El testamento más antiguo encontrado en la Santa Casa es el de un hombre soltero llamado Francisco Diaz, registrado el 22 de septiembre de 1632. En este documento se verifica el modo como un término clasificatorio podía ser sustituido sin tener su esencia alterada. Sus esclavas Leonor y Briey, descritas inicialmente como mulatas, serán luego mencionadas como negras.

Sobre la palabra "criollo", Antonio Moraes Silva escribió que se trataba del esclavo que nacía en la casa del amo; significado similar también al de animal, cría, que nacía "en nuestro poder". ${ }^{8}$ La ambigüedad tanto del color como la del nacimiento "local" se presenta desde el siglo XVII, pues en el testamento de Pe. Francisco d'Araujo aparece la información de que había dejado para la Santa Casa de la Misericordia a "Rufina, Criollita", así como "Cecilio criollo que dicen que vive fuera [liberto]". ${ }^{9}$ Es común encontrar en la historiografía el término criollo usado para el negro que nació en Brasil. ${ }^{10} \mathrm{Si}$ el uso común de criollo fue únicamente el de negro nacido en esas condiciones, como se explicaría el hecho de Diogo Fernandez registrara en 1632, que tenía un "mulato que nació en su casa, hijo de su negra de nombre Izabel (...)", ¿qué queda de los términos liberto y libre?.

El sentido dado al término "criollo" era menos exclusivo del negro "nacional" que una categoría social "de color" aplicado a los descendientes de esclavos, pero que también podía ser atribuido a aquellos esclavos venidos de una parte de África. Un dato que ratifica esa afirmación es del ya citado testamento de André Fernandes de Bastos y de su mujer Catherina de Azevedo, en cual los testadores registraban que poseían veinte y tres "piezas de esclavos de guinea, criollos, cuyos nombres son los siguientes (...)”. ${ }^{11}$ Sí en el primer ciclo de la esclavización "esclavos de Guinea"

\footnotetext{
${ }^{8}$ Dicionário da Lingua Portuguesa.

${ }^{9}$ Arquivo da Santa Casa da Misericòrdia da Bahia, Livro $2^{\circ}$ do Tombo, f.38.

${ }^{10}$ Aunque la literatura de los ochenta haya registrado esta aceptación, podemos ver en este periodo la indicación del término "criollito" con doble significado- color y nacionalidad. Ver Kátia de Queiròs Mattoso, "O filho da escrava (em torno da lei do ventre livre)", em Revista Brasileira de Historia vol.8, n. ${ }^{\circ}$ 16, São Paulo, Associação Nacional de História, 1988, pp.7-55.
}

${ }^{11}$ Arquivo da Santa Casa da Misericòrdia da Bahia, Livro $2^{\circ}$ do Tombo, 1652-1685, f.16. 
era una expresión genérica que englobaba esclavos de varias etnias, ${ }^{12}$ la designación "criollo" también podía indicar el esclavo que hablaba la lengua criolla.

Si el siglo XVII anuncia nuestra etnogénesis, el XVIII apuntó y consolidó uno de nuestros dilemas: la ambigüedad clasificatoria. La segunda mitad de este siglo revela una continuidad clasificatoria y trae nuevos significados, inclusive en otras capitanías. En 1772, la subdivisión poblacional de Piauí revela la existencia de nueve categorías: "blanco", "preto", "rojo" (indio de cualquier nación), "mulato", "mestizo", "mameluco", "caful", "cabra" e "curiboca". Si mulato tiene el mismo significado dicho anteriormente, "cabra" era aplicado al hijo de preto y mulato, así como "caful" designará el hijo de preto con indio. Lo que llama la atención es el uso del término "mestizo", el cual significa lo "que participa de blanco, preto e indio", o sea, una categoría que servía como un depósito de muchas mezclas, inclusive las de origen colonial de difícil discernimiento. Es fue lo que sucedió cuando el oidor de la capitanía, Antonio José de Morais Durao, al discutir sobre los tipos de humanos de la capitanía de San José del Piaú y sus villas, afirmó que "cuando no se pueden distinguir bien por sus muchas mezclas, se explica con la palabra mestizo, es lo que yo hago, comprendiendo en ella los cabras y curibocas", siendo estos últimos, al principio, los hijos de mestizos e indios. ${ }^{13}$ El mestizo se transformaba, por tanto, en una posible ilusión de sentidos, ya que se presentaba como aquel que es responsable de ser fruto de orígenes diversos.

Observando las designaciones presentes en la Santa Casa de la Misericordia en Salvador de Bahía encontramos el uso de categorías raciales para los africanos que andaban pari passu (en igualdad de condiciones) con la nación de origen. En los recibos de salida de los internados del hospital, se colocaba el nombre, la condición social, la residencia, el lugar de origen y el color: "Agostinha da Penha, criolla liberta habitante de la parroquia de Nuestra Señora de Santa Gloria”; Antonio da Trindade, preto liberto, natural de la isla de príncipe"; João de Moraes, hombre mestizo e liberto, natural de Cabo Verde"; Theodozio Pinheiro, hombre pardo, habitante del Cabulla, parroquia de San Antonio". ${ }^{14}$ Si estos ejemplos refuerzan el

\footnotetext{
12 Ver, por ejemplo, Luís Viana, O negro na Bahia, $3^{\text {a }}$ ed., São Paulo, Nova Fronteira, 1988. Para uma discussào sobre a imprecisào terminológica no período escravocrata, ver Maria Inés Cortes de Oliveira, "Quem eram os 'negros da Guiné’? A origem dos africanos na Bahia”, em Afro-Asia n. ${ }^{\circ} 19-$ 20, Salvador de Bahía, Universidade Federal da Bahia, 1997, pp.37-74.

${ }^{13}$ Luiz Mott Apud, Piaui Colonial. Populacho, economia e sociedade, Teresina, Projeto Pertronio Portella, 1985, pp.22 e ss.

${ }^{14}$ Arquivo da Santa Casa da Misericòrdia da Bahia, Recibos de Despesas, 1751-1752, f.2.
} 
argumento de que la Santa Casa de la Misericordia reproducía un sistema de clasificación presente en la sociedad brasileña, la multiplicidad de significados allí presentes es singular cuando se observa la Rueda de los Expósitos.

\section{2.- La clasificación en la Rueda de los Expósitos*}

La Rueda de los Expósitos (ver imagen 1), instituida en Salvador de Bahía en 1726, se asemejaba a la de Lisboa. Los niños allí dejados encontraban nodrizas dirigidas por una directora, cuya acción era fundamental en la identificación del niño o niña. Era ella quien examinaba y acordaba la hora de la exposición en la rueda, indicando al tesorero, responsable por el registro en el libro de los expósitos, el sexo, el color, los rasgos, ropas, y hasta las notas que frecuentemente acompañaban al recién nacido. ${ }^{15}$

El primero de mayo de 1766 se colocó en la rueda de los abandonados a una mulata, y trajo consigo un pañal de hilo de lino, cosido en un pedazo de lino azul, y blanco, y una camisa de paño de lino, ya roto, y en el cintillo una cinta desvanecida de seda. ${ }^{16}$

El 6 de noviembre de 1776, se colocó en la rueda a una niña, con categoría de cabra, y trajo consigo dos camisas, un pañal de bayeta fabricado de algodón, un cintillo, un abrigo típico bahiano, llamado paño da costa, que ya estaba viejo. ${ }^{17}$

\footnotetext{
* La "Rueda de los expósitos" consistía en un mecanismo giratorio situado en una de las ventanas o de las puertas de las casas de beneficencia, en la que se dejaban a los niños de pocos meses de edad, para que desde adentro fueran recogidos por los funcionarios de esas instituciones. Nota del traductor.

15 Ver Inventàrio da Criacáo dos Expostos do Arquivo Histórico da Santa Casa da Misericòrdia de Lisboa, [Lisboa], [s.n.], 1998; Maria Luíza Marcílio, História social da criança abandonada, São Paulo, Hucitec, 1998, p.146. La importancia de la posición de tesorero y del escribano, y em especial del cargo de probador son mostradas por Luís dos Santos Vilhena, A Bahia no século XVIII, Salvador, Itapua, 1969, p.125, quien al llamar la atención sobre la riqueza de la Santa Casa da Misericòrdia, decía que la elección de mayor representación excedía a los pretendientes de Senado y cámara. Como observo Russel-Wood, Fildagos e Filantropos, p.90, los miembros de la Santa Casa na Bahia eran propietarios de haciendas, de plantaciones y de ganados. La observación del color en el registro de los expuestos fue el sesgo de la hidalguía bahiana.

${ }^{16}$ Arquivo da Santa Casa da Misericordia da Bahia, Livro $4^{\circ}$ dos Engeitados, 1763-1770, f.120. los datos aquí trabajados tienen como punto de partida los años sesenta del siglo XVIII, pues los libros anteriores $\left(1^{\circ}\right.$ a $\left.3^{\circ}\right)$ se encuentran muy deteriorados em el archivo de la Santa Casa da Misericordia.

${ }^{17}$ Arquivo da Santa Casa da Misericórdia da Bahia, Livro $5^{\circ}$ dos Expostos, 1770-1777, f.321.
} 


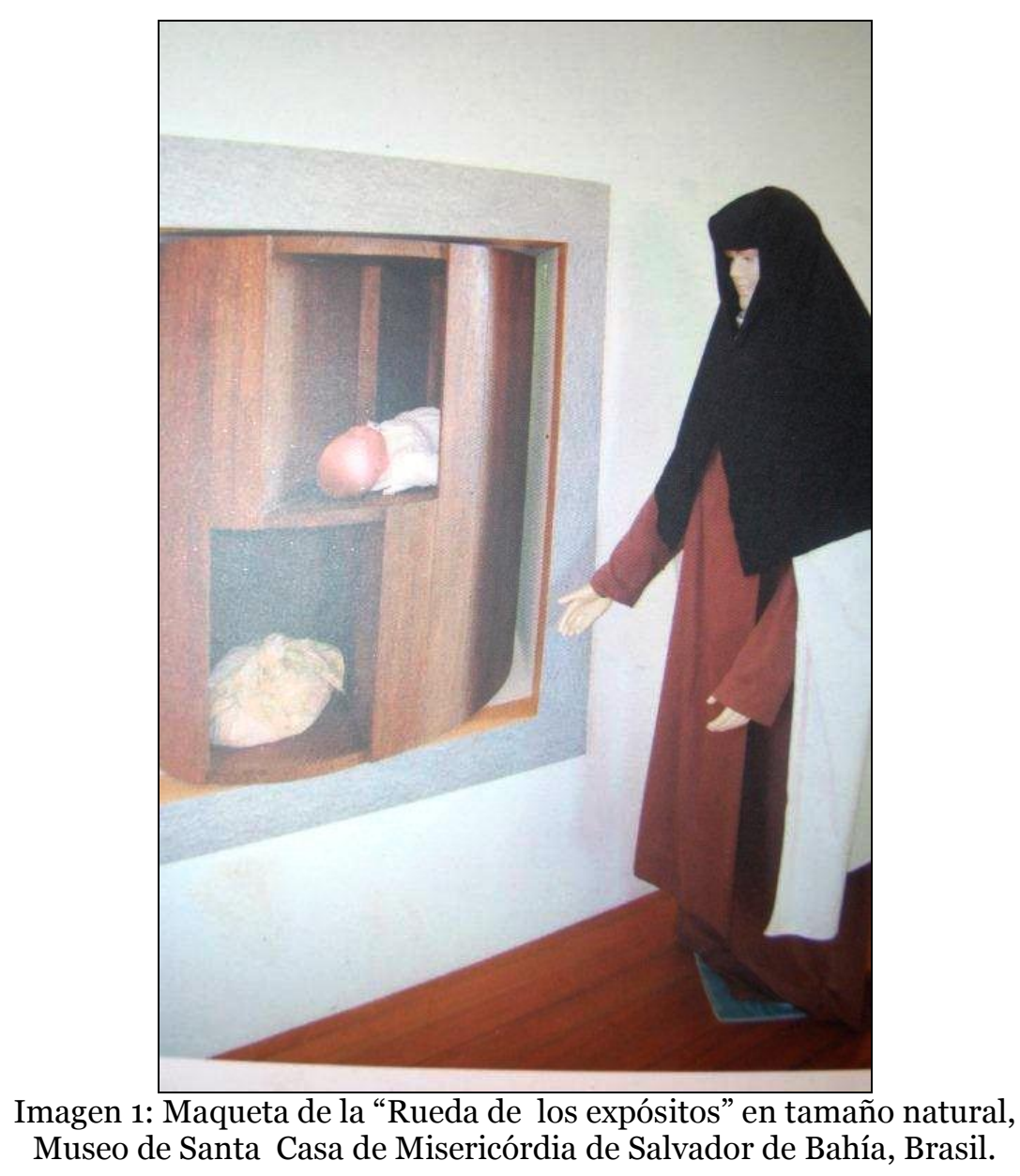

Los ejemplos dados nos muestran una precisión en los datos referentes al color y a las piezas de ropa, indicando los elementos principales en el momento del registro. Ciertamente, determinados objetos como los paños y los tejidos apuntaban un lugar social de los niños abandonados, lo que probablemente se transformaba en un elemento importante en la imaginación clasificatoria de la directora del lugar. $\mathrm{Nu}$ merosos fueron los pequeños criollos que llegaban con camisa de cambray de algodón, camisetas largas con calicó, cinturones rosa, o con gorras de calicó. Por lo tanto, pienso que la indumentaria es un elemento que no debe ser descartado en el sistema de clasificación del color de los abandonados. Se podría decir que la vestimenta ayuda en la definición de colores.

Sin embargo, considerando que la mayoría de los abandonados eran pobres, no debemos pensar que la indumentaria que traían indicaba una inmediata lectura 
de su clasificación racial, pues ropas viejas no eran una exclusividad de los mulatos, cabras o criollos pequeños, y una mayor cantidad de dichas piezas no se refería solamente a los blancos.

Muchas veces el registro de color no era fácil, como se verá en siguiente ejemplo: "El 12 de septiembre de 1776 se lanzó en la rueda un niño al parecer blanco, y trajo con el dos camisas, un pañal de bayeta roja, un paño de costal bahiano muy viejo, y en la cintura un cordón y en la cabeza un pañuelo". ${ }^{18}$

Varios son los ejemplos en los que se manifiesta la duda sobre el color de los niños abandonados. Es el caso de una niña que el 7 de abril de 1766 fue clasificada inicialmente como "blanca", pero al final del registro el escribano deja aparecer muestras de ambigüedad: "parecía ser una pequeña parda". ${ }^{19}$ ¿De qué forma el color blanco podría hacer dudar con el color pardo? Es posible pensar que distante de los colores socialmente construidos con base en la polaridad -preto o blanco-, la confusión se puede establecer cuando el color de los niños no se regulaba bajo esos patrones. Lo cierto es que podemos notar una terminología oficial en el Brasil colonial.

El término pardo, de acuerdo al diccionario de Moraes remite al "Color entre blanco y preto, como el del ave gorrión”, y también indica a un mulato, como el "aire pardo/marrón, el de mañana antes de esclarecer el día. Aún estaba el aire marrón, y esto ya empezaba a anochecer". ${ }^{20}$ Ahora, si a los ojos del escribano de la Santa Casa de la Misericordia el color de los niños podría ser más una indicación de las relaciones en que se desenvolvían pretos y blancos, deberíamos tener una categoría que apuntara a un distanciamiento lingüístico de los términos "preto" y "blanco". Me refiero a la categoría "pardo disfrazado" que aparece en los años setenta del siglo XVIII, aplicada a un "pequeño pardo disfrazado", entregado a la Sra. Anna da Conceicao, parda, soltera y habitante en N. Sr. de Nazareth". ${ }^{21}$

Hasta el final de los años setecientos e inicios de los ochocientos el sistema clasificatorio utilizado por la Santa Casa de la Misericordia de Bahía, recurría a un repertorio limitado de ocho categorías, incluyendo "pardo disfrazado" e "indio",

\footnotetext{
18 Arquivo da Santa Casa da Misericórdia da Bahia, Livro $5^{\circ}$ dos Expostos, 1770-1777, f.316, grifo meu.

${ }^{19}$ Arquivo de la Santa Casa de la Misericórdia de Bahia, Livro $4{ }^{\circ}$ dos Expostos, f.116.

${ }^{20}$ Dicionário da Lingua Portuguesa.

${ }^{21}$ Arquivo de la Santa Casa da Misericórdia de Bahia, Livro $6^{\circ}$ dos Enjeitados, 1770-1777, f.316.
} 
como podemos observar en la tabla 1 referente al periodo 1763-1805 algo que ya apuntaba una existencia de un sistema oficial de color. Los periodos de la tabla sigue el modo como están organizados los libros.

Tabla 1: Niños colocados en la ronda de los expósitos (1763-1805)

\begin{tabular}{|l|c|c||c|c|c|c||c|c||}
\hline \multirow{2}{*}{ Colores } & \multicolumn{2}{|c||}{$\mathbf{1 7 6 3 - 1 7 7 0}$} & \multicolumn{2}{|c|}{$\mathbf{1 7 7 0 - 1 7 7 7}$} & \multicolumn{2}{|c|}{$\mathbf{1 7 7 7 - 1 7 8 3}$} & \multicolumn{2}{|c|}{$\mathbf{1 7 9 6 - 1 8 0 5}$} \\
\cline { 2 - 10 } & Totales & $\mathbf{~ \% ~}$ & Totales & $\mathbf{\%}$ & Totales & $\mathbf{\%}$ & Totales & \% \\
\hline Blanco & 454 & 71,8 & 429 & 71,7 & 320 & 54,0 & 422 & 56,1 \\
\hline Pardo & 78 & 12,4 & 64 & 10,7 & 213 & 36,0 & 248 & 33,0 \\
\hline Mulato & 74 & 11,7 & 76 & 12,7 & 27 & 4,6 & 23 & 3,0 \\
\hline Cabro & 16 & 2,6 & 20 & 3,3 & 16 & 2,7 & 27 & 3,7 \\
\hline Criollo & 8 & 1,3 & 9 & 1,5 & 11 & 1,9 & 29 & 4,0 \\
\hline Mestizo & 1 & 0,1 & -- & -- & -- & -- & -- & -- \\
\hline Negro & 1 & 0,1 & -- & -- & 3 & 0,5 & -- & -- \\
\hline Parda disfrazada & -- & -- & 1 & 0,1 & 2 & 0,3 & -- & -- \\
\hline Caboclo & --- & -- & -- & -- & & -- & 1 & 0,1 \\
\hline Indio & -- & -- & -- & -- & -- & -- & 1 & 0,1 \\
\hline Total & 632 & 100,0 & 599 & 100,0 & 592 & 100,0 & 751 & 100,0 \\
\hline
\end{tabular}

Fuentes: Arquivo de la Santa Casa de Misericórdia de Bahia, Livro $4^{\text {s }}$ dos Expostos (17631770); Livro $5^{\mathrm{s}}$ dos Expostos (1770-1777); Livro 6 ${ }^{\mathrm{s}}$ dos Expostos (1777-1783); Livro $7^{\mathrm{s}}$ dos Expostos (1796-1805).

La idea de la existencia de un porcentaje mayor de blancos entre los abandonados en el periodo de los setecientos ya fue propuesta y discutida por RusselWood,22 indicando que había un número significativo de hijos ilegítimos abandonados por las familias blancas, una tendencia de las familias de los libertos absorbieron a los ilegítimos, o por la existencia de redes de compadrazgo, así como el hecho de que los niños "de color" nacidos de esclavas tendieron a quedar bajo el control del amo. ${ }^{23}$

\footnotetext{
22 A. J. R. Russel-Wood, Fidalgos e Filantropos, p.247.

${ }^{23}$ K. de Queiròs Mattoso, “Ofilho da escrava”, pp.39-40.
} 
No obstante, aun habiendo un limitado repertorio de colores, estaba la posibilidad de la duda en las clasificaciones. Dos ejemplos son significativos. El primero se refiere a un niño que fue llevado el 4 de octubre de 1783 a la Santa Casa de la Misericordia por una preta libre, llamada Thereza de Jesus María, el cual fue registrado por el escribano como "parece blanco". ${ }^{24}$ Este recurso aparece en algunos registros revelando una incertidumbre en el modo como atribuir a los niños un color. La duda clasificatoria demuestra una preocupación por no confundirse con los colores.

El segundo ejemplo demuestra que a finales de los años setecientos la preocupación por la clasificación precisa era constante. Y los celos por los colores también embargaban a quienes tenían que criar a los niños expósitos. El 21 de septiembre de 1792 una niña identificada como "mulata" fue entregada para ser criada a doña Ana Barbuda Lobo, esposa de Joaquim J. Coelho de Fonseca. Llama la atención el hecho de que doña Ana haya devuelto a la niña por ser blanca. El caso habría de tener un desdoblamiento, pues más allá de pedir un cambio "por haber ese día tomado un abandonado creyendo que fuese mulato, cuando la vio blanco lo mandó a cambiar por otra abandonada...”. Doña Ana cobraba las mesadas que le pagó la Santa Casa. ${ }^{25}$ En pocas palabras, en el registro se percibe una elección determinada por D. Ana, mulatica. ¿Cuál sería la razón de esta determinación? Sea cual sea que haya sido el motivo, aparecen las ganas de criar un niño de color.

Los años finales de los setecientos y las primeras décadas de los ochocientos apuntan una diferenciación social basada en caracteres biológicos que se relacionan en un mosaico de colores. Los niños abandonados llegaban con la marca de desprecio y de abandono, fuesen ellos de cualquier color. Entre tanto, si la atribución de color indicaba lecturas del espectro social, eso no significaba una mera conformación a las teorías racistas, como acontecerá en las subsecuentes décadas del siglo $\mathrm{xx} .{ }^{26}$ Lo más interesante es que la atribución de color dependía del momento de la

\footnotetext{
${ }^{24}$ Arquivo de la Santa Casa de la Misericordia de Bahia, Livro $7^{\circ}$ dos Expostos, 1796-1805, f.5.

${ }^{25}$ Arquivo de la Santa Casa de la Misericòrdia de Bahia, Livro $7^{\circ}$ dos Expostos, 1796-1805, f.28o.

${ }^{26} \mathrm{El}$ consenso aun existente entre los intelectuales es que el concepto de raza indica la existencia de herencias físicas permanentes entre los grupos humanos siendo en Brasil introducido a partir del final de la primera mitad del siglo XIX. Ver Lílian Schwartz, O espetáculo das raças, São Paulo, Cia. das Letras, 1993. Probablemente, debido a ese consenso, los estudios sobre los expósitos, tendieron a clasificar el color en categorías tales como los negros y los mestizos. Como resultado no se registró la riqueza terminológica y, mucho menos, se discute las razones para el aumento de la adopción de estas categorías. En este sentido véase, por ejemplo, Johildo Lopes de Athayde, "Filhos ilegítimos e crianças expostas (Notas para um estudo da família baiana no século XIX)”, em Revista da Acade-
} 
observación. Si en el inicio el examen lo hacía la jefe y era registrado por escrito por el tesorero, era en la confrontación posterior que los colores sociales adquirían e incorporaban otros significados sobre la observación del registrador. $\mathrm{Y}$ era en el momento de la confrontación que se revelaba un sistema clasificatorio en que las combinaciones entre los términos nos sorprenden. Los dos libros de las confrontaciones de los abandonados se refieren al periodo 1815-1832, y en ellos se presenta el momento de observación y análisis de los niños, algunos meses después de ellos haber sido entregados a las "amas de casas". El trámite seguido es el que definía la necesidad de confrontación:

Cuando se hacen los pagos de las mesadas, para tales abandonados, serán presentados infaliblemente para que se hiciesen las necesarias averiguaciones todas las veces que las Amas vengan a recibir el pago de la crianza de éstos y cualquier cambio por el curso del tiempo que hayan de tener en las características, se hará la competente declaración en su respectiva hoja que van numeradas y firmadas por mí, registrador actual de la mesa, para que no haya engaños, o dudas de ser el propio, que la casa alimenta, y para constar mande hacer estas reglas. Bahía, Secretaría de la Misericordia, og de agosto de 1815. Yo, Francisco Belens. 27

La confrontación era minuciosa pues no debía de haber espacio para las dudas en la identificación de los expósitos. El color y las características físicas como el cabello, boca, barbilla, rostro, cabeza, nariz y orejas componen un sistema de caracteres, cuyos términos, anteriormente asociados a la condición social, podrían adquirir otros sentidos. Es lo que acontece con una niña denominada Constancia, entregada a Manoel Pinto el 16 de agosto de 1814, a quien se le fue atribuida "ojos pardos" por el registrador.

mia de Letras da Bahia n. ${ }^{\circ}$ 27, Salvador de Bahía, Academia de Letras da Bahia, 1979, pp.9-25; Maria Luíza Marcílio, Historia Social da Criança; Andréa da Rocha Rodrigues, A infância esquecida: Salvador, 1900-1940, Dissertação de mestrado, Universidade Federal da Bahia, 1998.

${ }^{27}$ Arquivo de la Santa Casa de la Misericòrdia de Bahia, Livro $1^{\circ}$ das Confrontações dos Enjeitados, 1815-1824, f.2. 
Tabla 2: Confrontaciones de los abandonados, Santa Casa en Salvador (1815-1824)

\begin{tabular}{|l|c|c|}
\hline \multicolumn{1}{|c|}{ Colores } & Totales & Porcentajes \\
\hline Blanco & 189 & 37,3 \\
\hline Blanco alvo & 32 & 6,3 \\
\hline Blanco moreno & 20 & 3,9 \\
\hline Blanco bastante moreno & 4 & 0,8 \\
\hline Blanco bastante trigueiro & 3 & 0,6 \\
\hline Blanco trigueiro & 3 & 0,6 \\
\hline Blanco moreno macilento & 2 & 0,4 \\
\hline Blanco alvo y rosado & 2 & 0,4 \\
\hline Blanco bastante alvo & 2 & 0,4 \\
\hline Blanco y claro & -- & 0,2 \\
\hline Blanco claro y macilento & -- & 0,2 \\
\hline Blanco color pálida & -- & 0,2 \\
\hline Branco corado & -- & 0,2 \\
\hline Cabra & 30 & 5,9 \\
\hline Cabrinha escuro & -- & 0,2 \\
\hline Cabra y trigueiro & -- & 0,2 \\
\hline Cabra de color negro & -- & 0,2 \\
\hline Criollo & 11 & 2,2 \\
\hline Pardo & 158 & 31,2 \\
\hline Pardo claro & 16 & 3,2 \\
\hline Pardo alvo & 8 & 1,6 \\
\hline Pardo trigueiro & 5 & 1,0 \\
\hline Pardo bastante trigueiro & 4 & 0,8 \\
\hline Pardo y bastante alvo & 3 & 0,6 \\
\hline Pardo poco trigueiro & 2 & 0,4 \\
\hline Pardo escuro & 2 & 0,4 \\
\hline Pardo poco claro & 1 & 0,2 \\
\hline Pardo y bastante claro & 1 & 0,2 \\
\hline Pardo disfrazado & 1 & 0,2 \\
\hline Trigueiro & 1 & 0,2 \\
\hline Total & 507 & 100 \\
\hline Funte & 0,2 & \\
\hline
\end{tabular}

Fuente: Arquivo de la Santa Casa de Misericórdia de Bahia, Livro 1 $^{\text {s }}$ das Confrontações dos Enjeitados (1815-1824)

Lo más intrigante es la combinación de colores referida al color de la piel. Por ejemplo, Ana, la niña abandonada, registrada como "blanca morena", pero que en el acto realizado el 23 de enero de 1818 "se le reconoció ser clara" con ojos gran- 
des y "poco azules llegando a ser pardos", a pesar de "ser bastante morena". ${ }^{28}$ Anna, otra con el mismo nombre, entregada a una ama el 5 de enero de 1814, fue registrada en la confrontación como "blanca trigueña" o sea, tendría el color de trigo maduro, "llegando a moreno". 29

En algunos casos, el registrador resaltaba el termino trigueiro (trigueño). Trigueiro, no se confundía con pardo, pues una niña fue registrada como parda, pero en el acto realizado el 3 de noviembre de 1816 "se reconoció ser bastante trigueira". ${ }^{30} \mathrm{Al}$ subrayar el término trigueiro, el registrador indicaba un reforzamiento del sentido, así como su duda la resalta en el empleo de un determinado término y su substitución por otro escrito encima o por debajo. Es lo que se desprende del registro de un niño en el cual hay incertidumbre sobre su identificación como "pardo", y luego encima de esa palabra, se escribió "cabra”. La misma situación puede se observa con el niño João entregado a Joanna María, a quien la descripción inicial como "pardo" luego se le substituyó por el color "blanco". Para no provocar reparos el registrador afirmó: "En la averiguación que se hizo, se reconoce como blanco a este abandonado". ${ }^{31}$ Como se ve, en un momento el niño fue reclasificado hacia abajo y pasó de ser pardo a cabro, y luego fue promovido de pardo a blanco. Esto sugiere que en el inicio del siglo xIx pardo sería una palabra típica de "negociación racial”.

Pero la cuestión no era tan simple, la confrontación se tornaba posible para la exacerbación de nuestra multipolaridad racial secular, aunque predominaba desde lejos las categorías predominantes del sistema clasificatorio, en el caso blanco y pardo, ya que el rechazo de hijos por los negros era mínimo. En este periodo cabras, y principalmente blancos y pardos constituyeron un mosaico de colores que ganaban o perdían énfasis entre sus vecinos, como si los funcionarios de la Santa Casa buscasen desesperadamente un rigor descriptivo inconfundible. Es lo que se muestra en la próxima tabla.

\footnotetext{
${ }^{28}$ Arquivo de la Santa Casa de la Misericòrdia de Bahia, Livro $1^{\circ}$ das Confrontações dos Enjeitados, 1815-1824, f.3.

${ }^{29}$ Arquivo de la Santa Casa de la Misericòrdia de Bahia, , Livro $1^{\circ}$ das Confrontações dos Enjeitados, 1815-1824, f.14. El término "moreno" registrado por Antonio de Moraes Silva en el Dicionário da Lingua Portuguesa significaba "de color parda escura".

${ }^{30}$ Arquivo de la Santa Casa de la Misericòrdia de Bahia, , Livro $1^{\circ}$ das Confrontações dos Enjeitados, 1815-1824, f.198, grifado no original.

${ }^{31}$ Arquivo de la Santa Casa de la Misericòrdia de Bahia, , Livro $1^{\circ}$ das Confrontacòes dos Enjeitados,1815-1824, f.381.
} 
Tabla 3: Confrontaciones de los abandonados, Santa Casa en Salvador (1824-1832)

\begin{tabular}{|l|c|c|}
\hline \multicolumn{1}{|c|}{ Colores } & Totales & Porcentajes \\
\hline Blancos & 306 & 53,1 \\
\hline Pardos & 224 & 38,9 \\
\hline Criollos & 24 & 4,2 \\
\hline Cabras & 22 & 3,8 \\
\hline Total & 576 & 100,0 \\
\hline
\end{tabular}

Fuente: Arquivo de la Santa Casa de Misericórdia de Bahia, Livro $2^{\mathrm{s}}$ das Confrontações dos Enjeitados (1824-1832)

Tabla 4: Número de registros que muestran cambio en clasificación multirracial

\begin{tabular}{|l|l|c|}
\hline \multicolumn{1}{|c|}{$\begin{array}{c}\text { Clasificación del 1 } \\
\text { Registro }\end{array}$} & \multicolumn{1}{|c|}{$\begin{array}{c}\text { Clasificación en la } \\
\text { Confrontación }\end{array}$} & Total de casos \\
\hline Blanco & Blanco moreno & 3 \\
\hline Blanco & Pardo & 2 \\
\hline Blanca & Blanco bastante albo & 2 \\
\hline Blanco & Blanco y claro & 1 \\
\hline Blanco & Pardo y bastante claro & 1 \\
\hline Blanco & Branco y trigueiro & 3 \\
\hline Pardo & Blanco & 2 \\
\hline Pardo & Pardo albo & 3 \\
\hline Pardo & Cabra & 14 \\
\hline Pardo & Pardo y claro & 1 \\
\hline Pardo & Pardo y bastante trigueiro & 1 \\
\hline Pardo & Pardo y bastante escuro & 2 \\
\hline Pardo & Pardo bastante claro & 2 \\
\hline Crioulinha & Cabra & 1 \\
\hline Cabra & Cabra e clara & 1 \\
\hline Cabra & Criolla & 44 \\
\hline Total & & \\
\hline
\end{tabular}

Fuente: Arquivo de la Santa Casa de Misericórdia de Bahia, Livro $2^{\mathrm{s}}$ das Confrontações dos Enjeitados (1824-1832)

El primer libro nos permite verificar la existencia de treinta categorías multipolares, en un total de 507 niños registrados, e indica las amplias posibilidades del sistema clasificatorio. Son cuatro las categorías predominantes- "blanco", "cabra", "criollo" y "pardo" siendo que "blanco y "pardo" comprendían el 68,5\% de aquel total. Las categorías "alvo", "morenos", "claro", "trigueiro", y "escuro" son los términos de sentido agregado, pues pueden ser atribuidos a tres de las cuatro cate- 
gorías predominantes. Con excepción de "criollo", las demás categorías pueden componer cualquier repertorio en la clasificación.

Si en este libro está el registro de confrontación, el segundo libro presenta dos sistemas clasificatorios que pueden ser comparados. El primer libro posee una mayor variedad combinatoria, reuniendo 119 de los 507 expósitos. En cuanto que el segundo libro contempla 44 niños, de un total de 576 , que fueron clasificados de un modo múltiple. Al principio puede parecer que la razón para tal diferencia sea la forma de observar y analizar de cada registrador sobre el color de los abandonados. Sin embargo, se trata menos de una clasificación individual y, si, de lecturas de prácticas sociales. Cuando se contrasta la clasificación del primer registro y la clasificación de la confrontación se nota en el segundo libro las posibilidades del cambio o aumento de otras categorías.

\section{3.- La definición en la combinación lingüística-social}

Los datos presentados sugieren puntos distintos pero relacionados. El lenguaje simbólico contenido en la clasificación utilizada por los registradores de la Santa Casa de la Misericordia revela el empleo en el Brasil colonial de un modelo formal de categorías raciales creado en la metrópoli portuguesa (las categorías utilizadas para los expósitos en la Casa Santa de la Misericordia en Lisboa eran "preto", "pardo", "negro", "mulato" y "blanco"), pero del que se distanciaban los encargados de registrar los niños al elaborar un repertorio local de categorías ambivalentes. En el "inventario de la creación de los abandonados del archivo histórico de la Santa Casa de la Misericordia de Lisboa", siglos XVIII y XIX, se registra "pardo" como el color entre blanco y preto, blanco sucio, oscuro, piel oscura o triguiera, muy moreno, mulato claro o lo mismo que mulato. "Negro" es un calificativo para niños de color oscuro, parecido al árbol del ébano y al ámbar negro y que pertenece a la raza o a lo negro. Un hecho singular entre los registros de las dos Casa de expósitos es que en el periodo entre junio de 1780 y marzo de 1833, la Santa Casa de Lisboa separaba el registro de niños de color blanco, tanto en el libro de entrada, como en el de las "crianza de leche". Los registros de crianza de leche de los pardos y pretos se hacían en los libros del mismo, por comarca. 
Si como sugiere Homi Bhabha, ${ }^{32}$ la fijeza es un signo de la diferencia cultural/histórica/racial en el discurso del colonialismo, estamos delante de múltiples significados contenidos en una combinación de términos que apuntan hacia nuevas formas de construcción de alteridades en el ambiente colonial. Es posible argumentar que la construcción lingüística/social en la clasificación de los abandonados no estaba restringida a la observación de los expósitos de la Santa Casa, sino que también se encontraba disponible en otros ambientes del Brasil de la época. O sea, las percepciones sobre el color traducían tanto las jerarquías sociales como también revelaban ambigüedades en el modo como los individuos eran clasificados. 33

Una pista, entre otras que podemos seguir es la caracterización de "pardo disfrazado". La utilización de ésta categoría, ya acentuada en 1770, puede ser verificada, en el siglo siguiente, en otras regiones. En la correspondencia del capitán Mor de Sao Cristovao, Sergipe, al gobernador de Bahía, el 7 de mayo de 1825, el primero decía haber arrestado más allá de "muchachos blancos, pardos disfrazados y no de color fuerte". Como argumenta Luiz Mott, la línea entre las designaciones "pardo disfrazado" y "Blanco mesclado" o entre "pardo apretado" e "cabra", era bastante débil, indicando otros atributos tanto físicos (no solamente el color) y sociales (la identificación de parientes o la inserción en el mundo de los blancos) hacían parte del "modus operandi" de la clasificación colonial brasileña. ${ }^{34}$

Si esas afirmaciones son pertinentes para el universo de los adultos, ¿̇de qué modo ellas se entenderían a quienes se presentaran en la Santa Casa de la Misericordia? Al final, los abandonados eran recién nacidos, abandonados en la noche en la rueda, sin identificación paterna o materna y, en la mayoría de las veces, sin identificación inmediata con las relaciones sociales del mundo colonial de sus padres. Algunos volantes y cartas amarrados en un cordón en el cuello del recién nacido indicaba el lugar social de la madre o del padre. Y no era algo exclusivo de los clasificados como los blancos, ya que los pardos también llevaban esto en el cuello.

Había volantes que no dejaban pistas, como fue el caso de aquel encontrado junto a una niña: "Esta niña debe llamarse Amancia, debe se debe procurar que sea feliz, feliz quien presenta esta solicitud sobre ella de aquí a algún tiempo". La úni-

\footnotetext{
32 Homi Bhabha, O local da cultura, UFMG, 1998, p.105.

${ }^{33}$ Silvia Hunold Lara, Fragmentos setecentistas: escravidáo, cultura e poder na América portuguesa, Tese de livre-docencia, Universidade Estadual de Campinas, 2004, pp.156 e ss.

${ }^{34}$ Luiz Mott Apud, Sergipe del Rey. Populacao, economia e sociedade, Aracaju, Fundesc, 1986, p.54.
} 
ca pista no indica su lugar social, apenas su infelicidad: "una madre infeliz". "Ella nació el día 15 de octubre de $1872 "$.

Otros dejan ver una relación directa con alguien de la elite local. El día 02 de mayo de 1876 fue recogido un niño blanco, de ocho días de nacido, y con el siguiente volante: "Se pide que sea tratado este niño con todo cuidado y desvelo pues es hijo de familia importante y rica. Él ha de ser reclamado por su papá en tiempo competente, y se pagará todas las despensas con generosidad". ${ }^{35}$ Este niño blanco resultó de relaciones consideradas ilegítimas por la sociedad local, posiblemente involucrando a personas de las elites. Hay un caso interesante de un niño pardo, denominado posteriormente como Xavier Joaquim de Mattos, abandonado con el siguiente volante:

\begin{abstract}
Alguien que no puede tener en su compañía un hijo por motivos particulares, y además de eso por verse sin recursos, se resolvió dejarlo en esta Santa Casa declarando aun no haber sido bautizado y debe ser llamarlo Joaquim. Y como en cualquier tiempo sus padres lo pueden buscar cuando las conveniencias así lo permitan, lleva como señal para el reconocimiento una cinta amarilla atada a la pierna izquierda. Nació el día 22 de Agosto de 1872.36
\end{abstract}

¿Sería Joaquim hijo de una relación considerada racialmente ilegitima? Todo lo que sabemos es que él se quedó en la Santa Casa de la Misericordia hasta los 14 años. El volante más curioso fue escrito en francés. El 17 de octubre de 1866, un niño de color pardo fue puesto en la rueda y trajo la siguiente nota: "El pequeño niño es expósito en este asilo hoy 17 de octubre de 1866 porque su madre es muy pobre y el padre está muerto. El niño, está bautizado y se llama Emmanuel François. Compasión de él, muy queridamente la hija de caridad”. ${ }^{37} \mathrm{Si}$ el origen del niño es pobre, ¿quién habría sido el autor de la nota escrita en francés? Al final, el conocimiento de esa lengua era bastante restricto. Desafortunadamente solo restan especulaciones.

\footnotetext{
${ }^{35}$ Arquivo de la Santa Casa de la Misericordia de Bahia, Livro $6{ }^{\circ}$ da Roda dos Expostos, 1874-1877, f.93.

${ }^{36}$ Arquivo de la Santa Casa de la Misericordia de Bahia, Livro $6{ }^{\circ}$ da Roda dos Expostos, 1874-1877, f. 58 .

${ }^{37}$ Arquivo de la Santa Casa de la Misericòrdia de Bahia, Livro $2^{\circ}$ da Roda dos Expostos, 1865-1867, f.18.
} 
El pequeño número de notas encontradas poco revela sobre el origen social de la mayoría de los expósitos. Entre tanto, aun sin que tengamos las identificaciones del lugar ocupado por sus padres en la sociedad bahiana, pienso que no había una completa exención del observar de quien registraba el color de los niños. O sea, es posible pensar que la mente de quien registraba los expósitos fuese permeada también por la imaginación del color de los padres de los niños. Un aspecto importante es que otros elementos presentados en la descripción, como las características fenotípicas, no son el elemento definitivo de los colores sociales. O sea, los rasgos biológicos del racismo del siglo XIX -la boca, la forma de la nariz y de los labios como características físicas en la definición del color- son menos definitorios que los colores sociales, como podemos percibir en las definiciones de las cuatro categorías básicas de color:

Blanca

La abandonada Crescencia dada para criar a Rosa Maria de Viterbo el 29 de marzo de $1819 . .$. es blanca, cabeza larga, cabello castaño, frenta alta- ojos negros- nariz larga y afilada- boca pequeña- labios ordinarios- rostro y barbilla extendida. Orejas redondas y planas- muestra tener un mes de edad, y es flaca. ${ }^{38}$

Pardo

Es pardo - cabeza comprimida- cabello rizo y negro- frente alta- ojos proporcionados y negros-nariz comprimida y ancha- rostro redondo, boca, y labios proporcionados-orejas ordinarias, y comprimidas muestra tener un año de edad. Esta nutrido. ${ }^{39}$

Cabra

El abandonado dado para criar a Maria Francisca el 03 de marzo de 1819... es cabra- cabellos crespos- frente alta. Ojos negros y grandes- nariz pequeña y plana -boca proporcionada- labios finos- orejas pequeñas y planas- esta flaca y muestra tener un año de edad. ${ }^{40}$

Criollo

\footnotetext{
${ }^{38}$ Arquivo de la Santa Casa de la Misericòrdia de Bahia, Livro $1^{\circ}$ das Confrontações, 1815-1824, f.16o.

${ }^{39}$ Arquivo de la Santa Casa de la Misericòrdia de Bahia, Livro $1^{\circ}$ das Confrontações, 1815-1824, f.179.

40 Arquivo de la Santa Casa de la Misericòrdia de Bahia, Livro $1^{\circ}$ das Confrontações, $1815-1824$, f.59.
} 
El criollo, cabeza larga- cabello negro- frente larga- ojos ordinarios y negrosnariz larga, ancha- boca, y labios proporcionados rostro redondo e igualmente la barbilla- orejas pequeñas y con el ceño fruncido. ${ }^{41}$

Esos términos presentan características aproximadas. Entre las semejanzas compartidas por esos niños, todos tienen la cabeza larga, cabeza elevada, y ojos negros. La característica distintiva de la niña blanca en comparación con los otros niños (pardo, criollo y cabra) es el rasgo de la nariz, dicho como "nariz larga y fileña", lo que parecería ser un rasgo muy expresivo. Cuando se observan las características de los no blancos las diferencias se presentan en términos de "cabello crespo" (cabra), nariz ("nariz larga y ancha"- pardo; "nariz larga, un tanto mal acentuada en la punta- criollo; nariz pequeña, y plana"- cabra). El formato del rostro se aproximaría al blanco y cabra ("larga"), pardo y criollo ("redondo").

Aunque estos rasgos se presenten como diferenciadores entre los que se clasificaron arriba como blanco, pardo, criollo y cabra, el formato de la nariz de los abandonados no se presenta como el signo de distinción de la clasificación de color en la Bahía colonial. Cuando se observa en el libro de la confrontación la descripción de la forma de la nariz se vuelve en un elemento usado para cualquier de los colores sociales, puesto que es en el momento de la confrontación que la posibilidad de combinación entre categorías y la de su interrelación con otros rasgos fenotípicos se presentaba. Si Bernardinho, "dado a criar el 28 de marzo de 1820" fue definido como "blanco alvo", con "nariz fileña", y en el acto de 26 de julio del mismo año "se vio tener ojos pardos", otros dos niños fueron clasificados del siguiente modo: La primera, inicialmente, como "blanca", y posteriormente en la confrontación "blanca y clara" que tenía cabellos castaños y "nariz pequeña y ancha". La segunda, "blanca", y, en la confrontación, "parece ser blanca" teniendo la "nariz larga e ancha". ${ }^{42}$

La categoría "blanca morena" se combinaba en torno de una nariz "larga y un tanto chata", en el caso de Anna Joaquina, que tenía un "cabellos rizado y castaño" o la "nariz ancha" de Manuel, también de cabello castaño, así como Martinha,

\footnotetext{
41 Arquivo de la Santa Casa de la Misericòrdia de Bahia, Livro $1^{\circ}$ das Confrontações, 1815-1824, f.179.

42 Arquivo de la Santa Casa de la Misericòrdia de Bahia, Livro 2 das Confrontagoes, fs.186, 88 e 155 .
} 
una abandonada que tenía cabellos negros y "nariz ancha un tanta plana junto a los ojos, y mal acentuada en la punta". ${ }^{43}$

Lo mismo sucedía con los clasificados bajo el término de pardo. Un niño "pardo y claro" con "cabello rizado y negro" tenía el formato de nariz "larga, y fileña". Otra "parda poco clara", de "cabello rubio, y algo rizado", poseía una nariz fileña. En cuanto que una abandonada "parda disfrazada" tenía "cabello rizado castaño" y "nariz pequeña, y algo mal acentuada". En el registro de esta última realizado el 18 de agosto de 1817, "se pensó tener está abandonada de ojos pardos y algo grandes, labios gruesos, y barbilla con una pequeña división, y orejas grandes, y con huellas. ${ }^{44}$ La nariz larga, "ancha y mal acentuada en la punta", identificado en una niña "criolla", fue también descrito en relación a Domingo, clasificado como "pardo y claro". 45

La definición de un niño clasificado en un registro del 6 de agosto de 1824 indicaba como siendo "blanco, moreno, cabra, cabellos negro, ojos grandes y negros, labios finos, nariz pequeña y ancha. Boca pequeña, orejas pequeñas. Y con el ceño fruncido". Casi dos años después de ese registro se dice en el acto que tenía el cabello castaño y "amarillento" [rizado].

¿Qué proporciona el tono de la caracterización social, son menos los rasgos físicos como la nariz, color inscrita en lo social. Si no, entonces como entender que, el 7 de julio de 1824, un abandonado fue clasificado como un "pardo albo", que tenía nariz pequeña, ancha, y frente pequeña? Aun así que se diferencie con un rostro redondo, ojos negros y grandes y orejas grandes, lo que sobresale como diferencial es realmente su color. Es de ahí que resulta que un niño catalogado en la rueda como "pardo" había sido en la confrontación, el primero de julio de 1825, registrado como "blanco" teniendo una "nariz plana junto a los ojos". 46

Una situación similar se presentó cuando el 19 de enero de 1820 se definían los rasgos de un abandonado "criollo", dado para criar: "Este abandonado es criollo- cabeza larga- cabello negro- frente alta- ojos ordinarios y negros- nariz larga,

\footnotetext{
${ }^{43}$ Arquivo de la Santa Casa de la Misericòrdia de Bahia, Livro 2 das Confrontagoes, f.5o.

${ }^{44}$ Arquivo de la Santa Casa de la Misericòrdia de Bahia, Livro $11^{\circ}$ dos Expostos, 1813-1821, fs.14, $213,268$.

${ }^{45}$ Arquivo de la Santa Casa de la Misericòrdia de Bahia, Livro $11^{\circ}$ dos Expostos, 1813-1821, fs.382, 370 e 166.

${ }^{46}$ Arquivo de la Santa Casa de la Misericòrdia de Bahia, Livro 11 ${ }^{\circ}$ dos Expostos, 1813-1821, f.210.
} 
ancha y algo mal formada en la punta- boca, y labios proporcionados-rostro redondo- e igualmente la barbilla- orejas pequeñas, y con el ceño fruncidos". ${ }^{4}$

La nariz no se presenta como el único signo definidor de una categoría racial, pues los registros tanto un "criollo", cuanto un "cabra", nos presentan, respectivamente, como teniendo la nariz "larga y ancha" y "nariz pequeña y ancha", así como un "pardo" que el 8 de mayo de 1819, tenía "cabellos rizado y negro, nariz larga ancha, rostro redondo, frente alta"

\section{Conclusión}

El registro de color se muestra como un "sello" gramaticalmente inscrito en el propio sistema de relaciones socio-raciales. No es para nada que la categoría "criolla" se muestre ausente como posibilidad combinatoria. La puesta en funcionamiento de combinación de los colores omite términos como "preto" o "negro" y hacía del criollo el depósito de esas categorías. Por cierto las categorías "preta" o "negra" en la sociedad brasileña se refería al "africano" y al " negro esclavo"; sin embargo, algunos abandonados fueron clasificados de tal forma, como puede ser observado en el periodo 1763-1770 (un niño) y 1777-1783 (tres niños). Aunque los abandonados fueron niños sin ninguna indicación formal del lugar que sus padres ocuparon en la sociedad, la indicación de su color los remitía en un lugar socialmente preestablecido, pero responsable, quien sabe, de transformaciones. En este sentido, la observación del registrador en la confrontación de un expósito es ejemplar. Presentado como "blanco, cabeza grande poco cabello y algo rubio [...] es albo...", se verifica que él tenía "color tigueiro... después se reflexionó que con el tiempo cambiaría de color". ¿Al final, si un niño "blanco" se convertía "pardo claro", no podría de la misma forma pasar con un infante "blanco y rubio" fuese identificado, posteriormente, como "trigueiro"? Los caminos para nuestros dilemas contemporáneos de clasificación racial ya estaban siendo tocados.

\footnotetext{
${ }^{47}$ Arquivo de la Santa Casa de la Misericòrdia de Bahia, Livro 2 das Confrontagoes, f.81.
} 


\section{Bibliografía:}

\section{Fuentes primarias:}

Arquivo da Santa Casa da Misericórdia da Bahia:

Livro $1^{\circ}$ do Tombo (Escrituras, aforamentos e testamentos), 1629-1635.

Livro $2^{\circ}$ do Tombo, 1652-1685.

Livro $4^{\circ}$ dos Enjeitados, 1763-1770.

Recibos de Despesas, 1751-1752, Livros 4 ${ }^{\circ}, 5^{\circ}$ dos Expostos, 1770-1777.

Livro $6^{\circ}$ dos Enjeitados, 1770-1777.

Livro $7^{\circ}$ dos Expostos, 1796-1805.

Livro $1^{\circ}$ das Confrontações dos Enjeitados, 1815-1824.

Livros $2^{\circ}$ y $6^{\circ}$ da Roda dos Expostos, 1874-1877.

Livro 1, $2^{\circ}$ das Confrontações, 1815-1824.

Livro $11^{\circ}$ dos Expostos, 1813-1821.

Diccionario da Lingua Portugueza recopilado de vocabularios impressos até agora, e nesta segunda edicäo novamente emendado e muito acrescentado por Antonio Moraes Silva, Lisboa, Typographia Lacerdina, 1813.

\section{Fuentes secundarias:}

Bhabha, Homi, O local da cultura, UFMG, 1998.

Butler, Kim D., Freedom given. Freedoms won. Afro-Brazlians in post-abolition. Sâo Paulo and Salvador, New Jersey, Rutgers University Press, 1998.

Cortes de Oliveira, Maria Inés, “Quem eram os 'negros da Guiné’? A origem dos africanos na Bahia”, Afro-Asia, n. ${ }^{\circ} 19-20$ Salvador de Bahía, Universidade Federal da Bahia, 1997, pp.37-74.

Costa, Paulo Segundo da, Ações sociais da Santa Casa da Misericórdia, Salvador, Contexto \& Arte, 2001.

Guimaráes Sá, Isabel dos, A Misericórdia da Bahia. Quando o rico se faz pobre: misericórdias, caridade e poder no império portugués 1500-180o, Lisboa, Comissão Nacional para as Comemorações dos Descobrimentos Portugueses, 1997.

Guimaráes, Antonio Sérgio, Racismo e anti-racismo no Brasil, Sáo Paulo, Editora 34, 1999.

Hunold Lara Silvia, Fragmentos setecentistas: escravidão, cultura e poder na América portuguesa, Tese de livre-docencia, Universidade Estadual de Campinas, 2004.

Inventàrio da Criacáo dos Expostos do Arquivo Histórico da Santa Casa da Misericórdia de Lisboa, [Lisboa], [s.n.], 1998. 
Lopes de Athayde, Johildo, "Filhos ilegítimos e criancas expostas (Notas para um estudo da família baiana no século XIX)", Revista da Academia de Letras da Bahia n. ${ }^{\circ}$ 27, Salvador de Bahía, Academia de Letras da Bahia, 1979, pp.9-25.

Maggie, Ivonne, “'Aqueles a quem foi negada a cor do dia': as categorias cor e raça na cultura brasileira”, in Marcos C. Maio e Ricardo V. Santos (org.), Raça, Ciência e Sociedade, Rio de Janeiro, Fiocruz / Centro Cultural Banco do Brasil, 1996, pp.225-234.

Marcílio, Maria Luíza, História social da criança abandonada, Sao Paulo, Hucitec, 1998.

Monteiro, John M., “As 'raças' indígenas no pensamento brasileiro do imperio", in Marcos C. Maio e Ricardo V. Santos (org.), Raça, Ciência e Sociedade, Rio de Janeiro, Fiocruz / Centro Cultural Banco do Brasil, 1996, pp.15-22.

Montes, Maria Lúcia, Misericórdia, a força de um legado histórico: pesquisa realizada para a Santa Casa da Misericórdia da Bahia, Sáo Paulo, [s. n.], 2002.

Mott Apud, Luiz, Piaui Colonial. Populacho, economia e sociedade, Teresina, Projeto Pertronio Portella, 1985.

Mott Apud, Luiz, Sergipe Del Rey. Populacao, economia e sociedade, Aracaju, Fundesc, 1986.

Ott, Carlos, A Santa Casa da Misericórdia da Cidade do Salvador, Rio de Janeiro, MEC, 1960.

Queiròs Mattoso, Kátia de, "O filho da escrava (em torno da lei do ventre livre)", Revista Brasileira de Historia, vol.8, n. ${ }^{\circ} 16$, Sao Paulo, Associação Nacional de História 1988, pp.37-55.

Queiroz, Delcele M., Raça, género e educação superior, Tese de doutorado, Universidade Federal da Bahia, 2001.

Rocha Rodrigues, Andréa da, A infância esquecida: Salvador, 1900-1940, Dissertação de mestrado, Universidade Federal da Bahia, 1998.

Rodrigues, Esteves A. Neuza (org.), Irmàos da Santa Casa da Misericórdia da Bahia século XVII, Salvador, Santa Casa da Misericòrdia, 1977.

Russell-Wood, J. R., Fidalgos e Filantropos - A Santa Casa da Misericórdia da Bahia, 1550-1755, Brasília, UnB, 1981.

Santos Vilhena, Luís dos, A Bahia no século XVIII, Salvador, Itapua, 1969.

Schwartz, Stuart B., Segredos Internos. Engenhos e escravos na sociedade colonial, Sáo Paulo, Companhia das Letras, 1988. 
Schwartz, Lílian, O espetáculo das ragas, Sao Paulo, Cia. das Letras, 1993.

Seyferth, Giralda, "Construindo a nação: hierarquias raciais e o papel do racismo na política de imigração e colonização", in Marcos C. Maio e Ricardo V. Santos (org.), Raça, Ciência e Sociedade, Rio de Janeiro, Fiocruz / Centro Cultural Banco do Brasil, 1996, pp.41-58.

Viana, Luís, O negro na Bahia, $3^{\text {a }}$ ed., São Paulo, Nova Fronteira, 1988. 